Les directives sont des recommandations élaborées par des spécialistes et qui reposent sur des études épidémiologiques et cliniques. De ce fait, elles sont naturellement soumises à de permanentes modifications et s'appliquent aux populations prises en compte dans les études et aux critères d'évaluation définis dans celles-ci.

D'une part, ces dernières années, des études d'intervention ont permis de faire des découvertes essentielles pour la prévention des maladies cardiovasculaires, ce qui a eu pour conséquence une modification très rapide des directives: ainsi, pour les patients à haut risque, le taux cible de LDL-cholestérol a été abaissé pratiquement tous les deux ans. D'un autre côté, les études épidémiologiques durent très longtemps et ne sont pas spécifiquement axées sur la population suisse.

Connaissant ces faits et sachant qu'un renouvellement des directives existantes se dessine déjà, le comité de direction de la
Société Suisse de Cardiologie a décidé, avec la collaboration du Groupe de Travail Lipides et Athérosclérose (GSLA), au lieu d'élaborer ses propres nouvelles directives, de présenter et de commenter celles de l'IAS et de l'ESC. Ainsi, le médecin traitant peut librement choisir les recommandations qu'il préfère, et, par là, la base de données.

Les recommandations de l'IAS offrent l'avantage de reposer sur une base de données prospective et d'utiliser comme critère d'évaluation «les événements cardiovasculaires». Les recommandations de l'ESC sont faciles à utiliser et s'appuient sur de nombreuses données mais sont fondées sur le critère «mort d'origine cardiovasculaire», sont de type rétrospectif et s'appuient sur des données émanant de centres très divers.

Prof. Dr Peter Buser, Prof. Dr Dr h.c. Walter F. Riesen

\title{
Recommandations pour la prévention de l'athérosclérose
}

\author{
W. F. Riesen, R. Darioli, G. Noseda, O. Bertel, P. Buser*
}

Deutsch erschienen

in Nr. 22/2005

\footnotetext{
* Au nom des comités de direction du GSLA et de la SCC et avec la collaboration de la Société Suisse de Médecine Interne (SSMI), de la Société Suisse de Médecine Générale (SSMG) et de la Société Suisse d'Hypertension (SSH)
}

Correspondance:

Prof. Dr Dr h.c. Walter F. Riesen, Institut de chimie clinique et hématologie

\section{Préambule}

Dans les pays industrialisés, les maladies athéroscléreuses demeurent la principale cause de mortalité $[1,2]$; elles sont d'origine multifactorielle et souvent liées au mode de vie [3]. Leur prévention - un problème social et médical capital poursuit deux objectifs [4]: D'une part, elle vise à diminuer, dans la population générale, les facteurs de risque liés au mode de vie, essentiellement tabagisme, alimentation athérogène, excès de poids/obésité et manque d'activité physique. D'autre part, l'objectif est d'identifier, dans la population générale, les personnes qui sont exposées à un risque accru de maladies athéroscléreuses et de mettre en œuvre les interventions cliniques qui réduisent leur risque cardiovasculaire.

Le procédé d'identification des patients à risque et l'intervention clinique dans ce groupe cible ont beaucoup changé ces dernières années. Alors que jadis on attribuait une grande importance à l'évaluation des facteurs de risque pris individuellement (recommandations du Groupe de travail Lipides et Athérosclérose 1999 [5]), c'est l'évaluation quantitative du risque global qui est aujourd'hui au premier plan. A cet égard, les recommandations des diverses instances spé- cialisées sont les mêmes. Ce concept d'évaluation du risque global est étayé par les résultats, récemment publiés, de l'étude INTERHEART. Celle-ci a clairement montré que $90 \%$ des infarctus du myocarde pouvaient être prévus grâce à l'évaluation de neuf facteurs de risque [6].

Le traitement intensif des patients symptomatiques est également incontesté: au vu des données probantes, toutes les recommandations américaines, européennes et internationales stipulent que, chez les patients présentant une athérosclérose manifeste des artères coronaires, cérébrales ou périphériques, il faut abaisser le taux de LDL-cholestérol à moins de 2,6 mmol/l $[4,7,8]$. Pourtant, les résultats de l'étude EUROASPIRE montrent que ces recommandations ne sont pas suffisamment appliquées à l'hôpital et en clinique [9]. Il en va de même pour la réduction de la pression artérielle, qui, d'après l'étude EUROASPIRE, est elle aussi négligée.

Par contre, il n'y a pas de consensus à propos de l'évaluation quantitative du risque global chez les sujets asymptomatiques. Cela tient essentiellement à la situation des données scientifiques: Les études épidémiologiques prospectives qui sont à la base des calculs ne concernent qu'une tranche d'âge limitée (PROCAM [10] et Framingham [11]) et ne prennent en compte 
qu'un nombre insuffisant de femmes (PROCAM). Etant donné que chez les femmes les événements surviennent plus tardivement que chez les hommes, il faudra attendre quelques années pour que le nombre de ces informations soit suffisant. L'étude SCORE pare à cette situation [12], avec des données sur la mortalité, mais elle ne prend en compte que la prévention de la mortalité, et non pas celle de la morbidité.

\section{Points communs aux diverses recommandations}

Les diverses recommandation présentent beaucoup de points communs. Cela vaut surtout pour les patients symptomatiques qui doivent recevoir un traitement intensif. Mais les facteurs de risque qui sont à la base du calcul, sont également pratiquement les mêmes. Les recommandations de la Third Joint Task Force of European and other Societies on Cardiovascular Prevention in Clinical Practice [8], que nous appelons ici directives 3JE/ESC, se limitent au cholestérol total (HDLcholestérol), à l'abus du tabac, au sexe, à l'âge et à l'hypertension artérielle. L'ATP-III et les directives de l'IAS prennent en outre en compte le taux de LDL-cholestérol, celui de triglycérides et les antécédents familiaux d'infarctus du myocarde précoce.

Alors que dans les recommandations ATP-III et 3JE/ESC, le diabète de type 2 est considéré comme équivalent à la présence d'une maladie coronarienne, dans les recommandations de l'IAS il est seulement considéré comme un facteur de risque. Cependant, le concept fondamental d'évaluation du risque global est le même. Les valeurs cibles pour les patients à haut risque sont les mêmes. Les mesures thérapeutiques à mettre en œuvre pour atteindre ces valeurs cibles sont pratiquement les mêmes dans toutes les directives.

Les différences concernent donc essentiellement le mode de calcul et les bases sur lesquelles reposent les calculs du risque global. Etant donné que les données se modifient très rapidement sur

\section{Tableau 1}

Principales similitudes entre les directives 3JE/ESC et les directives de I'IAS.

- Raisons du développement d'une stratégie active de prévention pour la pratique clinique

Principaux objectifs

Choix des priorités

Recommandations pour la prévention secondaire

Stratification du risque cardiovasculaire dans le cadre de la prévention primaire sur la base de l'évaluation du risque global

- Critères d'évaluation du syndrome métabolique la base des études cliniques, les recommandations concernant la prévention se modifient aussi de plus en plus.

\section{Analyse critique des diverses recommandations}

Etant donné qu'il existe différentes recommandations pour favoriser le dépistage précoce et la prévention des maladies cardiovasculaires, le médecin praticien se trouve inévitablement confronté à plusieurs documents d'origine différente. Les critiques adressées aux diverses directives concernent donc essentiellement l'hétérogénéité des études qui les sous-tendent et l'absence de validation dans les populations dans lesquelles on les utilise.

En Suisse, on utilise surtout les recommandations publiées en 2003 sous l'égide de l'European Society of Cardiology (directives 3JE/ESC) [8] et les recommandations de l'International Atherosclerosis Society (IAS), également publiées en 2003 [4]. Nous allons les comparer ci-dessous et les analyser à l'aide d'une étude pratique avec des données suisses.

Comme le montre le tableau 1, les deux directives (3JE/ESC et directives de l'IAS) concordent bien, tant du point de vue de leur utilisation que du point de vue des objectifs dans le domaine de la prévention cardiovasculaire en pratique clinique. Il en va de même pour les situations à grande priorité et pour la prévention secondaire.

Dans le cadre de la prévention primaire, on ne s'appuie pas non plus, dans ces deux directives, sur l'évaluation isolée de certains facteurs de risque, mais sur l'évaluation du risque global. Soulignons enfin que le syndrome métabolique a récemment été inclus comme facteur de risque associé à une augmentation du risque cardiovasculaire, comme cela était déjà le cas dans les recommandations américaines de 2001 (NCEPATP-III) [13]. Heureusement, les cinq critères qui permettent d'identifier le syndrome métabolique sont les mêmes dans les trois directives citées.

Le tableau 2 décrit les principales différences entre les directives 3JE/ESC et celles de l'IAS. Du point de vue méthodologique, l'ESC détermine le risque de mortalité d'origine coronarienne pendant les 10 années suivantes en se fondant sur des études de cohortes, menées dans douze régions européennes différentes. A cet effet, on a recruté, entre 1972 et 1991, plus de 200000 personnes d'âge moyen, avec une mortalité cardiovasculaire de 7000 personnes [13].

Par contre, les directives de l'IAS s'appuient sur l'étude PROCAM. Cette étude a permis de 
suivre une cohorte de 5389 hommes d'âge moyen (35-65 ans), chez lesquels sont survenus 325 événements coronariens [10]. Bien que le grand nombre de patients et d'études dans les divers pays européens semble un avantage pour les directives 3JE/ESC, cet avantage est atténué par la grande hétérogénéité et par le fait que seule la mortalité cardiovasculaire a été prise en compte. Par contre, certains facteurs plaident certainement en faveur de l'étude PROCAM: la méthodologie de l'étude, ses objectifs et la fiabilité des données. Les publications très variées parues au cours des dix dernières années prouvent bien que cette étude prospective est le pendant européen de la Framingham Heart Study [11].

L'évaluation du risque de mortalité sur 10 ans selon le score «3JE/ESC» englobe cinq facteurs de risque cardiovasculaire et est utilisable pour les deux sexes (voir fig. 1). La stratification du risque comprend deux catégories de patients à risque: patients chez lesquels le risque sur 10 ans est $\geq 5 \%$ et patients chez lesquels le risque sur 10 ans est $<5 \%$. De son côté, le score PROCAM comprend huit facteurs de risque, dont les principaux sont le taux de HDL-cholestérol et le taux de triglycérides - deux facteurs de risque étroitement associés au syndrome métabolique.

Le score PROCAM n'a pas été développé spécifiquement pour être utilisé chez les femmes. Mais les auteurs des directives de l'IAS préconisent d'utiliser, chez les femmes ménopausées, le même tableau que chez les hommes et, chez les femmes non ménopausées, de diviser par quatre le risque calculé pour les hommes. Ces recommandations sont fondées sur l'analyse de l'étude PROCAM. La stratification du risque selon les directives de l'IAS définit trois catégories: haut risque (>20\%), risque intermédiaire (10-20\%) et risque faible $(<10 \%)$ pour les 10 années à venir.

La comparaison des valeurs prédictives pour l'identification des personnes à haut risque selon l'étude PROCAM (tab. 3) montre que l'emploi

\section{Tableau 2}

Principales différences entre les directives 3JE/ESC et les directives de I'IAS pour la prévention primaire.

\begin{tabular}{|c|c|c|}
\hline \multirow[t]{2}{*}{ Composante } & \multicolumn{2}{|l|}{ Directives } \\
\hline & 3JE/ESC & IAS \\
\hline $\begin{array}{l}\text { Base de données pour l'évaluation } \\
\text { du risque global (ERG) }\end{array}$ & $\begin{array}{l}\text { Etudes de cohortes (hommes et } \\
\text { femmes) dans } 12 \text { régions euro- } \\
\text { péennes }\end{array}$ & $\begin{array}{l}\text { PROCAM (étude de cohorte menée } \\
\text { à Münster pendant } 10 \text { ans chez } \\
\text { des hommes) }\end{array}$ \\
\hline $\begin{array}{l}\text { Nombre de facteurs de risque pour } \\
\text { l'ERG }\end{array}$ & 5 & 8 \\
\hline $\begin{array}{l}\text { Principaux critères d'identification } \\
\text { des patients à haut risque }\end{array}$ & $\begin{array}{l}\text { Risque de mortalité cardiovasculaire } \\
\text { sur } 10 \text { ans }\end{array}$ & $\begin{array}{l}\text { Risque d'événements coronarien } \\
\text { aigu sur } 10 \text { ans }\end{array}$ \\
\hline $\begin{array}{l}\text { Fourchette d'âge pour la stratifi- } \\
\text { cation du risque }\end{array}$ & $40-65$ ans & $35-65$ ans \\
\hline $\begin{array}{l}\text { Evaluation spécifique du risque } \\
\text { chez les femmes }\end{array}$ & Oui & Non \\
\hline $\begin{array}{l}\text { Niveaux pour la stratification du } \\
\text { risque dans le cadre de la préven- } \\
\text { tion primaire }\end{array}$ & $<5 \%, \geq 5 \%$ & $<10 \%, 10-20 \%,>20 \%$ \\
\hline $\begin{array}{l}\text { Objectifs thérapeutiques pour les } \\
\text { lipides }\end{array}$ & 2 niveaux & 3 niveaux \\
\hline $\begin{array}{l}\text { Taux de lipides justifiant un traite- } \\
\text { ment hypolipémiant }\end{array}$ & Sur la base du risque sur 10 ans & $\begin{array}{l}\text { Sur la base du risque sur } 10 \text { ans } \\
\text { et du nombre de facteurs de risque }\end{array}$ \\
\hline $\begin{array}{l}\text { Qualification pour un traitement } \\
\text { hypolipémiant }\end{array}$ & Plus restrictive & Moins restrictive \\
\hline
\end{tabular}

Tableau 3

Comparaison entre les directives 3JE/ESC et les directives de I'IAS, du point de vue des valeurs prédictives.

\begin{tabular}{llr} 
Paramètre & 3JE/ESC Régions européennes à faible risque & IAS \\
\hline Spécificité diagnostique & $88,4 \%$ & $94,5 \%$ \\
\hline Sensibilité diagnostique & $39,7 \%$ & $35,7 \%$ \\
\hline Valeur prédictive positive & $19,8 \%$ & $32,0 \%$ \\
\hline Valeur prédictive négative & $95,3 \%$ & $95,3 \%$ \\
\hline Efficience diagnostique & $85,1 \%$ & $90,5 \%$
\end{tabular}


Figure 1

Risque de maladies cardiovasculaires mortelles sur 10 ans dans les régions européennes à risque cardiovasculaire faible.

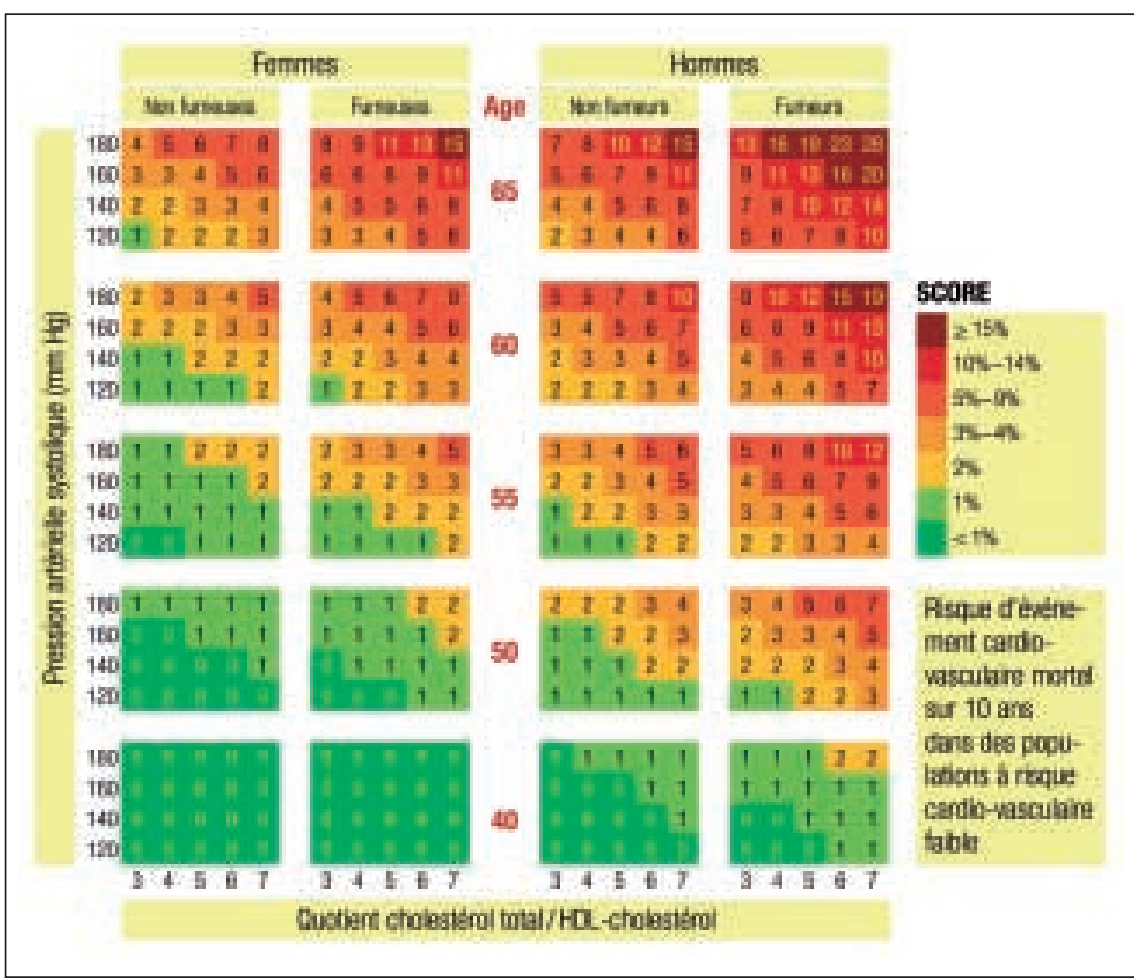

Figure 2

Score PROCAM: Risque d'événement coronarien aigu sur 10 ans chez les hommes et les femmes ménopausées. (Pour les femmes non ménopausées, il est recommandé de diviser par 4 le risque observé sur 10 ans chez les hommes de même âge; pour la population suisse, il est recommandé de corriger le risque sur 10 ans en le multipliant par le facteur 0,7 .)

\begin{tabular}{|lr|}
\hline Age (ans) & mint \\
\hline $35-39$ & 0 \\
\hline $40-44$ & $\frac{6}{11}$ \\
\hline $45-49$ & 16 \\
\hline $50-54$ & 21 \\
\hline $55-59$ & 26 \\
\hline $50-65$ & \\
\hline
\end{tabular}

\begin{tabular}{|c|c|}
\hline \multicolumn{2}{|c|}{$\begin{array}{l}\text { Preasion artilielle } \\
\text { systolique }\end{array}$} \\
\hline$<120$ & \\
\hline $120-129$ & 2 \\
\hline $130-139$ & \\
\hline $140-159$ & E \\
\hline$\geq 160$ & 8 \\
\hline
\end{tabular}

\begin{tabular}{|lr|}
\hline Diabète & ninh \\
\hline Dui & 5 \\
\hline Wini & Di \\
\hline
\end{tabular}

\begin{tabular}{|c|c|}
\hline LOL-C & Pints \\
\hline$<2,59$ & 0 \\
\hline $250-3,35$ & 5 \\
\hline $3,37-4,13$ & 10 \\
\hline $4,14-4,91$ & 14 \\
\hline$>4,91$ & 20 \\
\hline
\end{tabular}

\begin{tabular}{|c|c|}
\hline $\begin{array}{l}\text { Tabeglame } \\
\text { f12 enrian nety }\end{array}$ & hant \\
\hline Ou & 6 \\
\hline $\mathrm{Non}$ & 0 \\
\hline 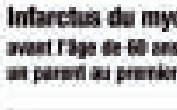 & $\begin{array}{l}\text { Carde } \\
\text { cher } \\
\text { devit } \\
\text { Parta }\end{array}$ \\
\hline DI & 4 \\
\hline Non & 0 \\
\hline
\end{tabular}

\begin{tabular}{lr}
\hline HOL-C & \\
mosire & Ponts \\
\hline c0.91 & 11 \\
\hline $0.91-1,15$ & 8 \\
\hline $1,17-1,41$ & 5 \\
\hline$>1,42$ & 0 \\
\hline
\end{tabular}

\begin{tabular}{|c|c|}
\hline 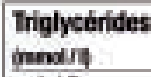 & Points \\
\hline$<1,15$ & 0 \\
\hline $1,15-1, \sqrt{2}$ & 3 \\
\hline $1,72-2,3$ & 3 \\
\hline$>2.3$ & 4 \\
\hline
\end{tabular}

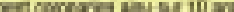

\begin{tabular}{|c|c|c|c|c|c|c|c|}
\hline Soove & $\%$ & Soome & 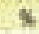 & Scone & $\leqslant$ & Soere & \\
\hline$<20$ & $<10$ & at & $2 \mathrm{~B}$ & \&1 & 70 & 51 & 16 \\
\hline $2 t$ & $1, t$ & 2 & 29 & 4 & 7,4 & 12 & e \\
\hline$n$ & 12 & 39 & 13 & 43 & $B b$ & 5 & 19 \\
\hline$n$ & 13 & 94 & 15 & 4 & as & Ba & 21 \\
\hline 24 & 14 & $\mathbf{5}$ & 4D & 46 & 102 & 35 & $n$ \\
\hline 25 & If & 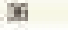 & 42 & 40 & 10.5 & 50 & 2 \\
\hline 26 & $t$ & $\pi$ & 48 & ar & 107 & $5 T$ & 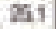 \\
\hline 27 & 18 & 39 & 5.1 & 48 & 12.8 & BA & 29 \\
\hline 28 & 19 & 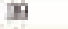 & 5) & 89 & 132 & 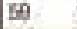 & 29 \\
\hline 29 & 23 & 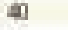 & E.1 & 0 & tas & 210 & \\
\hline & $2 i$ & & & & & & \\
\hline
\end{tabular}

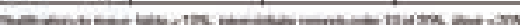

des recommandations de l'IAS permet d'opérer une meilleure distinction. En pratique, cela signifie une meilleure spécificité et donc une meilleure valeur prédictive positive, avec une valeur prédictive négative similaire pour les deux directives.

Le tableau 4 représente les différences du point de vue de la qualification d'un patient et des valeurs cibles pour un traitement hypolipémiant, d'après les recommandations 3JE/ESC et celles de l'IAS. Alors que l'ESC définit deux catégories, l'IAS en définit quatre, selon le risque absolu de présenter un événement coronarien au cours des dix années suivantes et en fonction du nombre de facteurs de risque cardiovasculaire associés. Il existe en outre des différences notables sur le plan des taux de lipides au-delà desquels il faut envisager un traitement médicamenteux hypolipémiant et aussi du point de vue des taux cibles de LDL-cholestérol qu'il faut atteindre, en fonction des catégories de risque.

L'IAS utilise le même procédé que celui des recommandations américaines que nous connaissons déjà bien grâce aux publications scientifiques parues depuis 1989, date de la première parution. Remarquons à ce propos qu'un procédé analogue a été utilisé lors de l'élaboration des recommandations par le GSLA [5]. Dans un deuxième temps, notre analyse concerne les conséquences de l'utilisation des diverses directives.

Les directives ont été utilisées sur une population de près de 9000 sujets asymptomatiques, âgés de 20 à 80 ans (âge moyen $45 \pm 12$ ans), qui ont été examinés dans le cadre du «Lausanne Health Promotion Program», comme cela a été récemment décrit [13]. Pour résumer, disons que chez tous les participants inclus dans ce programme entre 2001 et juin 2004, les facteurs de risque cardiovasculaire ont été systématiquement évalués selon les recommandations du GSLA. Pour l'évaluation du risque selon le score PROCAM, on a procédé à un ajustement (multiplication par 0,7$)$ pour tenir compte de la situation en Suisse (cet ajustement était justifié par une simulation effectuée à l'aide des données MONICA [(V. Wietlisbach †]). Pour les femmes, on a suivi les recommandations des auteurs de l'étude PROCAM.

Le tableau 5 montre clairement que la stratification du risque diffère beaucoup d'une directive à l'autre. Quelles que soient les directives utilisées, la proportion de patients définis comme «à haut risque» est très faible ( $2 \%$ contre $1 \%)$, mais la classification du risque selon les recommandations de l'IAS, pour la qualification en vue d'un traitement, semble mieux correspondre au risque sous-jacent. 
Comme autre différence importante, il faut citer la proportion de patients éligibles pour un traitement médicamenteux. Cette proportion est globalement de $2 \%$ si l'on utilise les directives 3JE/ESC, contre $12 \%(\mathrm{p}<0,001)$ si l'on utilise celles de l'IAS. Pour terminer, constatons que, même s'il existe une concordance satisfaisante pour les patients éligibles pour un traitement médicamenteux hypolipémiant (72-89\%), la concordance entre les deux procédés, évaluée par le test non paramétrique kappa, est particulièrement faible, dans la mesure où ce coefficient atteint au mieux 0,22.

Globalement, on peut conclure, d'une part, que la comparaison entre les directives élaborées par des sociétés spécialisées internationales montre une bonne concordance de points de vue pour ce qui est de la nécessité et des objectifs de la prévention cardiovasculaire. D'un autre côté, cette comparaison révèle les principales différences sur le plan de leur utilisation chez les personnes asymptomatiques, tant du point de vue de la stratification du risque que sur le plan de la qualification d'un patient pour un traitement médicamenteux.

Cette analyse indique la nécessité d'adapter les recommandations pour chaque pays, en tenant compte des données épidémiologiques spécifiques et des aspects cliniques et sociodémographiques et du système de santé. En outre, les difficultés rencontrées lors de l'élaboration des recommandations pour la prévention cardiovas- culaire soulignent la nécessité d'une structure qui puisse assurer la surveillance d'une maladie, l'un des défis majeurs du système de santé.

\section{Discussion}

La prévention des maladies athéroscléreuses poursuit deux objectifs: d'une part, elle vise à réduire les facteurs de risque liés au mode de vie, essentiellement tabagisme, alimentation athérogène, excès de poids/obésité et manque d'activité physique. L'autre objectif est d'identifier, dans la population globale, les personnes exposées à un risque accru de maladies cardiovasculaires et de mettre en œuvre les interventions cliniques qui permettent de réduire leur risque cardiovasculaire.

Pour la prévention des événements cardiovasculaires chez les patients présentant une athérosclérose préexistante (prévention secondaire), l'utilité clinique, mais aussi économique d'un traitement par des hypolipémiants et des antihypertenseurs est démontrée. C'est pourquoi, pour les patients qui ont une athérosclérose cliniquement patente, le seuil d'intervention doit être bas. Compte tenu de ces données claires, les directives 3JE/ESC et celles de l'IAS concordent dans la mesure où, chez les patients présentant une athérosclérose manifeste des artères coronaires, cérébrales ou périphériques, il faut diminuer le taux de LDL-cholestérol à moins de

\section{Tableau 4}

Comparaison entre les directives 3JE/ESC et celles de I'IAS du point de vue de la stratification du risque et du traitement des dyslipidémies dans le domaine de la prévention primaire.

\begin{tabular}{|c|c|c|c|}
\hline \multicolumn{4}{|l|}{ Directives 3JE/ESC } \\
\hline Risque sur 10 ans & $\begin{array}{l}\text { Recommandations concernant } \\
\text { le mode de vie }\end{array}$ & $\begin{array}{l}\text { Taux de lipides justifiant un traitement } \\
\text { médicamenteux }\end{array}$ & Objectifs du traitement \\
\hline$<5 \%$ & Oui & $\mathrm{CT} \geq 8,0 \mathrm{mmol} / \mathrm{l}$ ou $\mathrm{LDL}-\mathrm{C} \geq 6,0 \mathrm{mmol} / \mathrm{l}$ & $\mathrm{CT}<5,0 \mathrm{mmol} / \mathrm{l}$ et $\mathrm{LDL}-\mathrm{C}<3,0 \mathrm{mmol} / \mathrm{l}$ \\
\hline$\geq 5 \%$ & Oui & $\mathrm{CT} \geq 5,0 \mathrm{mmol} / \mathrm{l}$ ou $\mathrm{LDL}-\mathrm{C} \geq 3,0 \mathrm{mmol} / \mathrm{l}$ & $\mathrm{CT}<4,5 \mathrm{mmol} / \mathrm{l}$ et $\mathrm{LDL}-\mathrm{C}<2,5 \mathrm{mmol} / \mathrm{l}$ \\
\hline \multicolumn{4}{|l|}{ Directives de I'IAS } \\
\hline Catégorie de risque & $\begin{array}{l}\text { Recommandations concernant } \\
\text { le mode de vie }\end{array}$ & $\begin{array}{l}\text { Taux de lipides justifiant un traitement } \\
\text { médicamenteux }\end{array}$ & Objectifs du traitement \\
\hline $\begin{array}{l}\text { Haut risque (risque de maladie } \\
\text { coronarienne sur } 10 \text { ans }>20 \% \text { ) }\end{array}$ & Oui & $\mathrm{LDL}-\mathrm{C} \geq 2,6 \mathrm{mmol} / \mathrm{I}$ & $\mathrm{LDL}-\mathrm{C}<2,6 \mathrm{mmol} / \mathrm{I}$ \\
\hline $\begin{array}{l}\text { Risque modérément augmenté } \\
\text { ( } \geq 2 \text { FR et risque de maladie } \\
\text { coronarienne sur } 10 \text { ans compris } \\
\text { entre } 10 \text { et } 20 \% \text { ) }\end{array}$ & Oui & LDL-C $\geq 3,4 \mathrm{mmol} / \mathrm{I}$ & $\mathrm{LDL}-\mathrm{C}<3,4 \mathrm{mmol} / \mathrm{l}$ \\
\hline $\begin{array}{l}\text { Risque intermédiaire ( } \geq 2 \text { FR et } \\
\text { risque de maladie coronarienne } \\
\text { sur } 10 \text { ans }<10 \% \text { ) }\end{array}$ & Oui & LDL-C $\geq 4,1 \mathrm{mmol} / \mathrm{I}$ & $\mathrm{LDL}-\mathrm{C}<3,4 \mathrm{mmol} / \mathrm{I}$ \\
\hline $\begin{array}{l}\text { Risque faible }(0-1 \mathrm{FR} \text { et risque } \\
\text { de maladie coronarienne } \\
\text { sur } 10 \text { ans }<10 \%)\end{array}$ & Oui & LDL-C $\geq 4,9 \mathrm{mmol} / \mathrm{l}$ & $\mathrm{LDL}-\mathrm{C}<4,1 \mathrm{mmol} / \mathrm{I}$ \\
\hline
\end{tabular}


2,6 mmol/l. Cependant, les résultats de l'étude EUROASPIRE montrent [9] que ces recommandations ne sont pas suffisamment appliquées en milieu hospitalier et en clinique.

Alors que les directives 3JE/ESC et celles de l'IAS sont très concordantes du point de vue de la prévention secondaire et des mesures thérapeutiques à prendre à ce propos, elles diffèrent du point de vue de l'évaluation du risque chez les patients asymptomatiques ou présymptomatiques. Les deux recommandations préconisent certes l'évaluation du risque global, mais la méthode de calcul et les études qui sous-tendent le calcul sont différentes.

Les directives 3JE/ESC sont fondées sur des données rétrospectives concernant la mortalité, alors que les recommandations de l'IAS s'appuient sur les données de l'étude PROCAM, une étude prospective. Comme la Suisse est un pays à risque relativement faible, les données de l'étude PROCAM ne peuvent pas être appliquées telles quelles à la Suisse. On peut certes adapter les données à la situation qui prévaut en Suisse en utilisant l'étude MONICA, mais cela ne vaut que pour les cantons de Vaud, Fribourg et le Tessin. Nous manquons de données suffisamment fiables à propos de la morbidité et de la mortalité cardiovasculaires reposant sur des études épidémiologiques concernant l'ensemble de la Suisse.
Contrairement aux directives de l'IAS, dans le calcul du risque global les directives 3JE/ESC ne tiennent comptent ni des antécédents familiaux de maladies cardiovasculaires, ni du taux de triglycérides. Elles utilisent en outre le taux de cholestérol total au lieu du taux de LDL-cholestérol, lequel est cependant considéré comme la cible thérapeutique. En outre, le taux de HDLcholestérol est négligé, bien que dans le projet SCORE on ait aussi élaboré des tableaux pour le quotient cholestérol total/HDL-cholestérol.

Comme valeur seuil pour l'instauration d'un traitement médicamenteux, les directives $3 \mathrm{JE} / \mathrm{ESC}$ préconisent un risque de mortalité cardiovasculaire $\geq 5 \%$ sur 10 ans, ce qui correspondrait à un risque de morbidité cardiovasculaire de $20 \%$ en l'espace de 10 ans. Il existe en outre des différences entre les deux directives sur le plan de la qualification des patients pour un traitement médicamenteux. Etant donné ces différences pour ce qui est de la stratification pour la prévention primaire, le nombre de patients justiciables d'un traitement est également différent.

On peut aussi prévoir que les nouvelles études, actuellement en cours, fourniront de nouvelles données, en particulier sur les valeurs cibles, ce qui aura pour conséquence l'élaboration de nouvelles recommandations. Pour les patients à risque particulièrement élevé, l'IAS a déjà fixé de

Tableau 5

Comparaison entre les directives 3JE/ESC et celles de I'IAS du point de vue du traitement des dyslipidémies en prévention primaire. Etude de 8829 personnes dans le cadre du «Lausanne Health Promotion Program».

\begin{tabular}{|c|c|c|c|c|c|c|}
\hline \multicolumn{7}{|l|}{ Directives ESC } \\
\hline \multirow[t]{2}{*}{ Risque sur 10 ans } & \multicolumn{3}{|c|}{ Pourcentage (\%) } & \multicolumn{3}{|c|}{ Qualification pour un traitement hypolipémiant } \\
\hline & Femmes & Hommes & Tous & Femmes & Hommes & Tous \\
\hline$<5 \%$ & $3652(99,8 \%)$ & $5057(97,8 \%)$ & $8709(98 \%)$ & $37-3652(1 \%)$ & $54-5057(1 \%)$ & $91-8709(1 \%)$ \\
\hline$\geq 5 \%$ & $7(0,2 \%)$ & $113(2,2 \%)$ & $120(2 \%)$ & $7-7(100 \%)$ & $100-113(89 \%)$ & $107-120(89 \%)$ \\
\hline Total & $3659(100 \%)$ & $5170(100 \%)$ & $8829(100 \%)$ & $44-3659(1 \%)$ & $154-5170(3 \%)$ & $198-8829(2 \%)$ \\
\hline \multicolumn{7}{|l|}{ Directives de I'IAS } \\
\hline \multirow[t]{2}{*}{ Catégorie de risque } & \multicolumn{3}{|l|}{ Proportion (\%) } & \multicolumn{3}{|c|}{ Qualification pour un traitement hypolipémiant } \\
\hline & Femmes & Hommes & Tous & Femmes & Hommes & Tous \\
\hline $\begin{array}{l}\text { Haut risque (risque de maladie } \\
\text { coronarienne sur } 10 \text { ans } \geq 20 \% \text { ) }\end{array}$ & $22(0,6 \%)$ & $65(1,3 \%)$ & $87(1 \%)$ & $22-22(100 \%)$ & $65-65(100 \%)$ & $87-87(100 \%)$ \\
\hline $\begin{array}{l}\text { Risque modérément augmenté } \\
(\geq 2 \text { FR et risque de maladie } \\
\text { coronarienne sur } 10 \text { ans compris } \\
\text { entre } 10 \text { et } 20 \%)\end{array}$ & $108(2,9 \%)$ & $239(4,6 \%)$ & $347(4 \%)$ & $96-108(89 \%)$ & $234-239(85 \%)$ & $300-347(86 \%)$ \\
\hline $\begin{array}{l}\text { Risque intermédiaire ( } \geq 2 \mathrm{FR} \text { et } \\
\text { risque de maladie coronarienne } \\
\text { sur } 10 \text { ans }<10 \% \text { ) }\end{array}$ & $435(11,9 \%)$ & $1688(32,6 \%)$ & $2123(24 \%)$ & $86-435(20 \%)$ & $352-1688(21 \%)$ & $438-2123(21 \%)$ \\
\hline $\begin{array}{l}\text { Risque faible }(0-1 \text { FR et risque } \\
\text { sur } 10 \text { ans }<10 \%)\end{array}$ & $3094(84,6 \%)$ & $3178(61,5 \%)$ & $6272(71 \%)$ & $77-3094(2,5 \%)$ & $158-3178(5 \%)$ & $235-6272(4 \%)$ \\
\hline Total & $3659(100 \%)$ & $5170(100 \%)$ & $8829(100 \%)$ & $281-3659(8 \%)$ & $779-5170(15 \%)$ & $1060-8829(12 \%)$ \\
\hline
\end{tabular}


Tableau 6

Concordance entre les directives 3JE/ESC, celles de I'IAS et celles du GSLA/de la SSC.

\begin{tabular}{|c|c|c|c|c|c|c|c|c|c|c|c|}
\hline \multirow{3}{*}{ 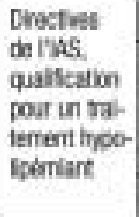 } & \multicolumn{3}{|c|}{ 3.E-ESC } & \multirow{3}{*}{ 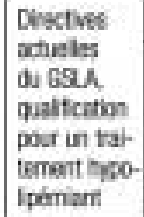 } & \multicolumn{3}{|c|}{ 3.E.ESC } & \multirow{3}{*}{$\begin{array}{l}\text { Directives } \\
\text { achueles } \\
\text { du GSEA, } \\
\text { qualfarion } \\
\text { pour un trai- } \\
\text { temant typo- } \\
\text { ipeniant }\end{array}$} & \multicolumn{3}{|c|}{ WS } \\
\hline & \multicolumn{2}{|c|}{$\begin{array}{c}\text { Qualfcation } \\
\text { pour un trabment } \\
\text { typolpemiant }\end{array}$} & \multirow[b]{2}{*}{ Totas } & & \multicolumn{2}{|c|}{$\begin{array}{l}\text { Oualfcation } \\
\text { pour in tatement } \\
\text { hpolgomiart }\end{array}$} & \multirow[b]{2}{*}{ Tale } & & \multicolumn{2}{|c|}{$\begin{array}{l}\text { Oud frarbon } \\
\text { pur un tatement } \\
\text { inpol pomant }\end{array}$} & \multirow[b]{2}{*}{ Total } \\
\hline & Ou & $\operatorname{lon}$ & & & Dus & Nan & & & Oui & Non & \\
\hline Du & 152 & 908 & 1050 & ou & 56 & 1976 & 2032 & Ouf & 319 & 741 & 1060 \\
\hline Non & 46 & 723 & 7768 & $\operatorname{Non}$ & 12 & 6656 & 6797 & $\mathrm{man}$ & 1713 & 0065 & 7789 \\
\hline Total & 196 & E631 & 8Rzg & Total & 198 & B631 & 8829 & Totril & 2032 & 6787 & 5929 \\
\hline \multicolumn{3}{|c|}{ Oualicerbos ESC } & $2,0 \%$ & \multicolumn{3}{|c|}{ Oatufcabon ESC. } & 2,08 & \multicolumn{3}{|l|}{ Qual fresbon US } & $12,0 \%$ \\
\hline \multicolumn{3}{|c|}{ Dualication LS } & $12,0 \%$ & \multicolumn{3}{|c|}{ Quaffcabon GSA } & 23.08 & \multicolumn{3}{|c|}{ Gidforosn GSLA } & $230 \%$ \\
\hline \multicolumn{3}{|l|}{ Concortince } & 89,28 & \multicolumn{3}{|l|}{ Concentance } & $\pi 6.04$ & \multicolumn{3}{|l|}{ Concrintancer } & 72.27 \\
\hline \multicolumn{3}{|c|}{ Concontance darpis } & $08,3,6$ & \multicolumn{3}{|c|}{ Concentatce Eีerpe } & $75,0 \times 6$ & \multicolumn{3}{|c|}{ Concundarce derpe } & $70,5 x$ \\
\hline \multicolumn{3}{|l|}{ Keqpa } & 0,22 & \multicolumn{3}{|l|}{ Keqpe } & 0,01 & \multicolumn{3}{|l|}{ Kepse } & 0,05 \\
\hline \multicolumn{3}{|l|}{ Vacur $\phi 80$} & $=0.000$ & \multicolumn{3}{|l|}{ Gabur dop } & $=0,04$ & \multicolumn{3}{|l|}{ Vabs dip } & $=0.000$ \\
\hline
\end{tabular}

nouvelles valeurs cibles pour le taux de LDL-cholestérol [14].

Etant donné les faits et les prévisions susdécrits, les représentants du GSLA ont décidé, en accord avec le comité de direction de la Société Suisse de Cardiologie, de promouvoir la prévention secondaire et de discuter des directives de l'ESC et de l'IAS pour la prévention primaire. Mais il faut laisser les médecins libres de leur choix - en sachant que l'on s'efforce déjà d'aboutir à des recommandations communes pour toute l'Europe au cours des années à venir.

\section{Références}

1 Murray CJ, Lopez AD. Mortality by cause for eight regions of the world: Global Burden Disease Study. Lancet 1997;349:1269-76.

2 Lopez AD, Murray CJ. The global burden of disease, 1990-2020. Nat Med 1998;4:1241-3.

3 Carleton RA, Dwyer J, Finberg L, Flora J, Goodman DS, Grundy SM, et al. Report of the Expert Panel on Population Strategies for Blood Cholesterol Reduction. A statement from the National Cholesterol Education Program, National Heart, Lung and Blood Institute, National Institutes of Health. Circulation 1991;83:2154-232.

4 International Atherosclerosis Society. Harmonized guidelines on prevention of atherosclerotic cardiovascular diseases. Full report. www.athero.org, April 30, 2003.

5 Battegay E, Bertel O, Darioli R, Gutzwiller F, Keller U, Nigg C, et al. Recommandations 1999 pour l'indication au traitement du facteur de risque «cholestérol». Bull Méd Suisses 2000; 81:2144-9.

6 Yusuf S, Hawken S, Ônpuus S, Dans T, Avezum A, Lanas $\mathrm{F}$, et al. Effect of potentially modifiable risk factors associated with myocardial infarction in 52 countries (the INTERHEART study). Lancet $2004 ; 364: 937-52$.
7 Executive Summary of the Third Report of The National Cholesterol Education Program (NCEP) Expert Panel on Detection, Evaluation, and Treatment of High Blood Cholesterol in Adults (Adult Treatment Panel III). JAMA 2001;285:2486-97.

8 DeBacker G, Ambrosioni E, Borch-Johnson K, Brotons C, Cifkova R, Dallongville J, et al. European guidelines on cardiovascular disease prevention in clinical practice. The Third Joint Task Force of European and other Societies on Cardiovascular Disease Prevention in Clinical Practice (constituted by representatives of eight societies and by invited experts) executive summary. Eur Heart J 2003;24:1601-10.

9 Anonymous. Clinical reality of coronary prevention guidelines: a comparison of EUROASPIRE I and II in nine countries. EUROASPIRE I and II Group. European Action on Secondary Prevention by Intervention to Reduce Events. Lancet 2001; 357:995-1001.

10 Assmann G, Cullen P, Schulte H. Simple scoring scheme for calculating the risk of acute coronary events based on the 10-year follow-up of the prospective cardiovascular Münster (PROCAM) Study. Circulation 2002;105:310-5.

11 Wilson PW, D'Agostino RB, Levy D, Belanger AM, Silbershatz H, Kannel WB. Prediction of coronary heart disease using risk factor categories. Circulation 1998;97:1837-47.

12 Conroy RM, Pyörälä K, Fitzgerald AP, Sans S, Menotti A, De Backer G, et al; SCORE Project group. Estimation of ten-year risk of fatal cardiovascular disease in Europe: the SCORE project. Eur Heart J 2003;23:987-1003.

13 Prior JO, van Melle G, Crisinel A, Burnand B, Cornuz J, Darioli R. Evaluation of a multicomponent worksite health promotion program for cardiovascular risk factors - correcting for the regression towards the mean effect. Prev Med 2005;40:259-67.

14 Grundy SM, Cleeman JI, Merz NB, Brewer BB Jr, Clark LT, Hunninghake DB. NCEP report: implications of recent clinical trials for the National Cholesterol Education Program Adult Treatment Panel III guidelines. Circulation 2004;110:227-39. 